\title{
A circulatory ionic wind for inertial sensing application
}

\author{
Ngoc Tran Van, Tung Thanh Bui, Thien Xuan Dinh, Canh-Dung Tran, Hoa Phan-Thanh, Trinh Chu \\ Duc, Van Thanh Dau
}

\begin{abstract}
A novel gyroscope using circulatory electrohydrodynamics flow in a confined space is presented for the first time. The configuration of the new gyroscope includes three pointring corona discharge actuators which generate ion flows in three separated sub-channels. The three ion flows then merge together when going through a nozzle of the main chamber entrance and create a jet flow. In the new configuration, the residual charge of ion wind flow is removed by a master-ring electrode located at one end of the main chamber. Under the effect of the angular speed of gyroscope, the jet flow is deflected and this deflection is sensed using hotwires. Results, which are consistently acquired by both numerical simulation and experiment on our prototypes, demonstrate the repeatability and stability of the new approach. Since the ion wind can be generated by a minimum power, the present configuration-based device does not require any vibrating component. Thus, the device is robust, cost and energy-effective.
\end{abstract}

Index Terms - Angular velocity, Corona, Gyroscopes, Numerical simulation.

\section{INTRODUCTION}

$\mathrm{E}$ LECTRO-HYDRODYNAMIC (EHD) flow, also called ion wind, with many advantages including lower cost and energy consumption, tidy and light but solid structure and simple operation is considered as a potential method in various applications and supported by recent technological advances in microfabrication [1]. The approach based jet flow has been recently applied in airflow control [2], propulsion technology [3] and bio-electronic device [4]-[6]. In parallel, several other techniques were also developed to generate jet flow in a confined space for the fluidic gyroscope, such as the vibration by a lead zircona-titanate (PZT) diaphragm [7], [8]; the electrokinetic using electro-conjugate fluid [9] and the thermal convection from a locally heated region [10]. In spite of efforts, those techniques are either instable while working with a linear acceleration or complicated in design [11]. The ion wind corona discharge can be applied to develop angular rate sensors, where a flow is able to freely vibrate in three-dimensional space by the inertial force. However, when the ion wind circulates inside a hermitical space, the residual charge yields a reversed electrical

N.V. Tran: Institute of Missile - Military Institute of Science and Technology, Vietnam

T.T. Bui: VNU University of Engineering and Technology, Hanoi, Vietnam (tungbt@vnu.edu.vn)

T.X. Dinh: School of Science and Engineering, Ritsumeikan University, Shiga 525-8577, Japan (thien@cfd.ritsumei.ac.jp)

C.-D. Tran: School of Mechanical and Electrical Engineering, University of Southern Queensland, Queensland QLD 4350, Australia (CanhDung.Tran@usq.edu.au) field which damages the corona discharge process. Although the use of ion wind in angular rate sensors was mentioned in several intellectual disclosures [12]-[14], its technical documentation was not sufficiently presented.

In this work, a novel design for ion wind generating device is proposed and applied in angular rate sensing. For this approach, a closed system based on ion wind principle with multiple point-ring electrodes is developed for inertial sensing applications. A master ring is introduced into the new system to neutralize the residual charge. The present device is firstly simulated for an initial design and possible estimation of the system characteristics. A gyroscope prototype is then manufactured using commercially available components.

The evaluations with regard to the mentioned advantages of corona discharge jet flow are also carried out while studying the properties of the present gyroscope prototype.

\section{MECHANISM AND EXPERIMENTAL SET-UP}

The main component of the present gyroscope is a fluidic network consisting of three ion wind cylindrical channels with dimension $5 \mathrm{~mm} \times 10 \mathrm{~mm}$ (diameter $\times$ length) each. The channels connect together before being linked to a working chamber (dimension: $12 \mathrm{~mm} \times 15 \mathrm{~mm}$ ) by a nozzle at the device center where hotwires are installed. One corona actuator with a pair of stainless steel SUS304 pin-ring is installed in each ion wind channel (see Fig. 1(a)). By an applied high voltage, charged ion winds are generated in channels and then propagate toward the nozzle where they merge together to create a jet flow moving through the working chamber. The ion jet flow then circulates back to each and every wind channels to be accelerated. A cylindrical master-ring with $10 \mathrm{~mm}$ diameter is set-up at the downstream end of working chamber to eliminate the residual charge of ion winds and hence stabilize ion flow while the corona is discharging. An image of sensor prototype is given in Fig. 1(c).

When the gyroscope is subjected to an angular rate, an induced Coriolis force deflects ion winds in the working

H. Phan-Thanh: Institute of Technology, Hanoi University of Industry, Vietnam

C.D. Trinh: University of Engineering and Technology, Vietnam National University, Hanoi, Vietnam (trinhcd@ vnu.edu.vn)

V.T. Dau: School of Engineering and Built Environment, Griffith University, Queensland QLD 4222, Australia (v.dau@griffith.edu.au) 
(a)

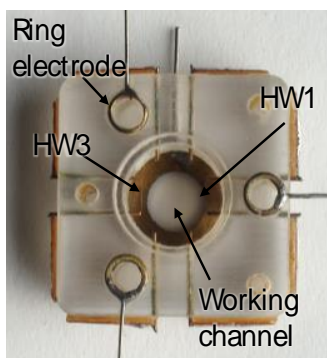

(c)

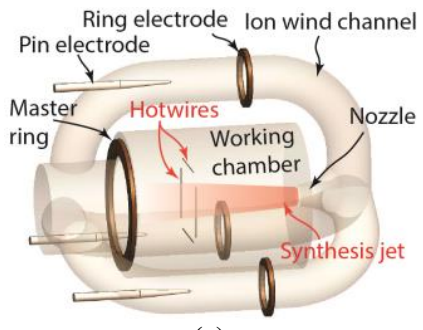

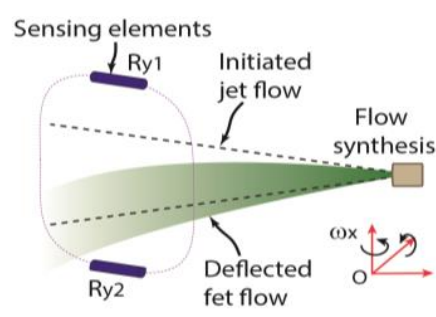

(b)

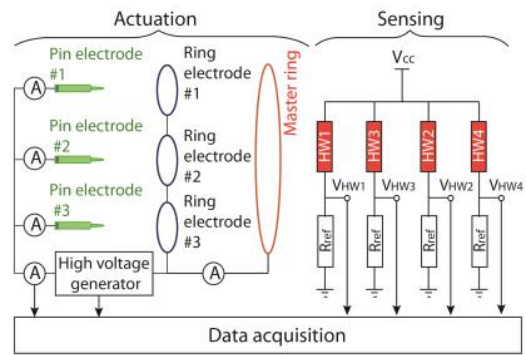

(d)
Fig. 1. Mechanism of a jet flow based gyroscope. (a) Hotwire arrangement is to detect the jet flow deflection under the rotations about $\mathrm{x}$ - and $\mathrm{y}$-axis; (b) Deflection of generated jet flow by the effect of angular velocity; (c) an experimental prototype of the sensor; and (d) measurement circuit.

chamber as schematized in Fig. 1(b). In order to detect this deflection, four tungsten hotwires are symmetrically installed surrounding the centerline of working chamber at a distance of $2.5 \mathrm{~mm}$ in a plane located $15.0 \mathrm{~mm}$ away from the nozzle as shown in Fig. 1(a).

Hotwires are connected to an electric circuit integrated into the device to capture signals by the voltage variations measured on them using NI9234 data acquisition module with a sampling rate of $25.6 \mathrm{kHz}$ and NI Signal Express-data logging software (National Instrument Ltd). In this work, Glassman EH10R10 to apply high voltages between pin-ring electrodes and an amperemeter M244T41 with scale of $10 \mu \mathrm{A}$ to measure the current variation of the system are used. The measurement circuit is presented in Fig. 1(d).

\section{RESULTS AND DISCUSSION}

The current-voltage (I-V) characteristics of each and the whole three pairs of pin-ring electrodes are determined using a measurement circuit as shown in Fig. 1(d). The I-V relationships of corona discharge depict the discharge current increases with the increase of applied voltage. However, while the relationships measured on the three pairs of pin-ring electrodes are nonlinear and nearly the same (Fig. 2(a)), the I$\mathrm{V}$ characteristics of the whole system is quasi-linear as observed in Fig. 2(a)-(b). Moreover, experimental observations in Fig. 2(b) found that an additional discharged current induced by the ion wind when the master ring is activated by connecting with ground. In fact, the gradient of I-V curve of the grounded master ring is steeper than one of the floating master ring. This depicts a significant effect of the grounded master ring on the I$\mathrm{V}$ characteristics of each pair of ping-ring electrodes and then the whole system. Experimental results by Fig. 2(b) show an increase of more than $25 \%$ for the discharge current when the
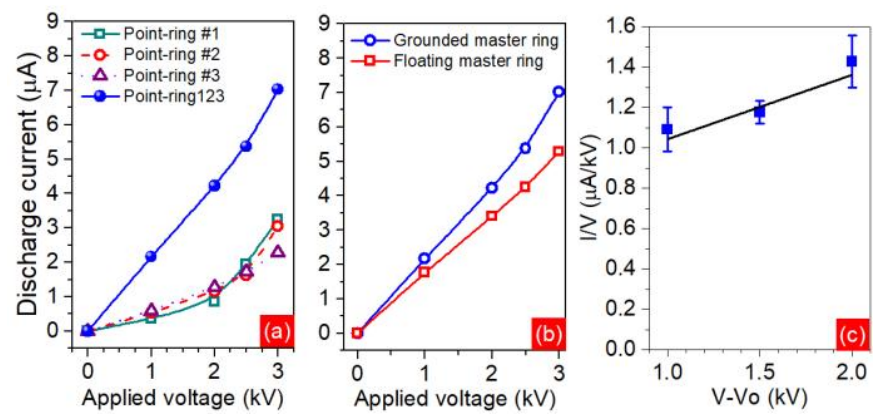

Fig. 2. Current-Voltage (I-V) characteristics of the system: (a) I-V characteristics of the whole system and the three pairs of pin-ring electrodes with grounded master ring; (b) I-V characteristics of the whole system with floating master and grounded master rings; and (c) I-V characteristics of the three pairs of pin-ring electrodes with grounded master ring by the empirical Townsend relationship.

master-ring is in-used.

The I-V characteristics of the new system is also stydied using the empirical Townsend relationship $(\mathrm{I} / \mathrm{V} \propto \mathrm{V})$. This method was initially applied in the coaxially cylindrical configuration. The approach was then developed for several other electrode configurations including the point-to-ring [14] as $\mathrm{I}=A \times \mathrm{V}\left(\mathrm{V}-V_{0}\right)$, where $\mathrm{I}$ is the discharge current; $\mathrm{V}$ the applied voltage; $V_{O}$ the inception voltage for the corona; and $A$ coefficient depending on the electrode geometry and ion mobility $(\mu)$. The I-V characteristics by Townsend relationship of the present configuration is presented in Fig. 2(c) with a coefficient $A=0.383 \mu \mathrm{A} / \mathrm{kV}^{2}$.

A numerical analysis of the circulatory EHD flow in the present gyroscope is also carried out. The simulation is a multiphysical problem related to (i) the electrical field which induces the migration of ions within the inter-electrode region; (ii) the interaction between ion winds and air flow in working channel; and (iii) the motion of air flow in the gyroscope. Under the effect of electric field, ion wind generated from pin electrodes moves and then drifts air flow which, in turn, is redistributed itself across the domain under consideration.

The electric field $\vec{E}$ is governed by the Poisson equation as $\nabla \cdot \overrightarrow{\mathrm{E}}=-\nabla^{2} \mathrm{~V}=\mathrm{q} / \epsilon_{0}$, where $\epsilon_{0}$ is the permittivity of free space and $q$ the total charge.

The corona discharge is set up as the boundary condition on electrodes' surfaces. Assume that the charge density $q_{s}$ on electrodes' surface is a function of the discharge current I as $q_{s}=\mathrm{I} /\left(\mu E_{\mathrm{on}} A\right)$, where $A$ is the total area of electrodes from which the electric field is generated with a magnitude greater than the onset electric field $E_{\text {on }}=3.23 \times 10^{6} \mathrm{~V} / \mathrm{m}$ [15]; and the discharge $I$ is referred from the I-V characteristics (Fig. 2). Neglecting the permittivity gradient, di-electrophoretic and electrostriction forces, only the Coulomb force $f_{e}\left(f_{e}=q \vec{E}\right)$ by the electromagnetic field is introduced into Navier-Stokes equation in considering the incompressible air flow.

Simulation results confirm the flow generated by the corona actuator, which is circulated back to electrode pins through the three subsidiary channels. The ion flow keeps its jet shape after getting out of the nozzle. 

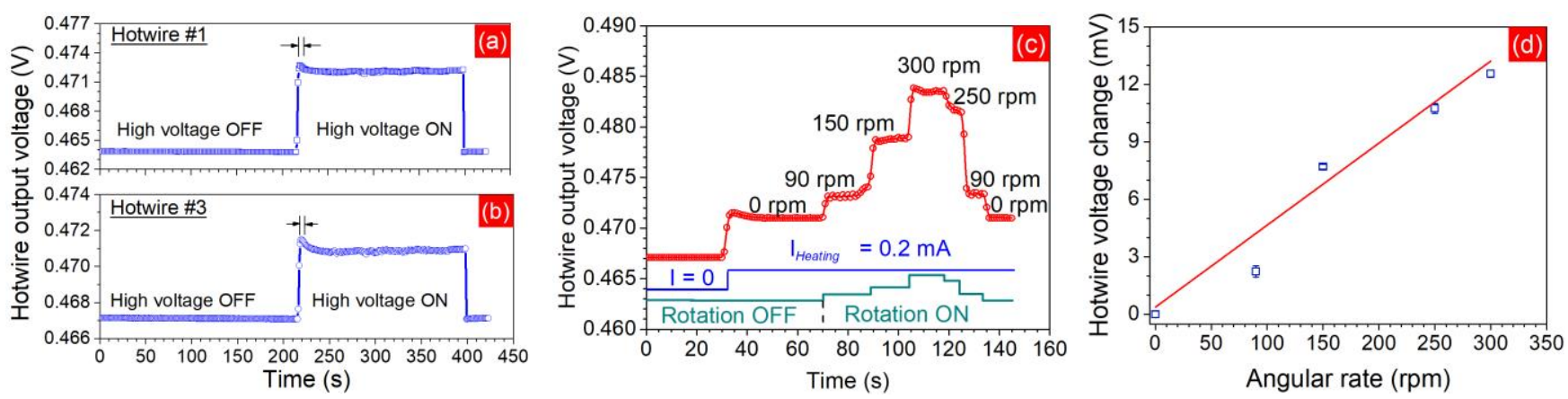

Fig. 3. Experimental results: (a-b) Time revolution of output voltage measured on hotwire 1 and hotwire 3 before and after applying high voltage, (c) the angular rate sensing by hotwire output voltage at different angular rates and (d) output voltage measured on hotwires versus the turntable's angular speed.

In a point-ring configuration, the velocity $U$ of ion wind is estimated from the discharge current $\mathrm{I}$ as $\mathrm{U}=k \sqrt{\mathrm{I} / \rho \mu}$ [15] where $k$ depends on the electrode discharge area and the interelectrode distance; $\mu=1.6 \times 10^{-4} \mathrm{~m} 2 \cdot \mathrm{V}^{-1} \cdot \mathrm{s}^{-1}$ the ion mobility; $\rho$ $=1.2041 \mathrm{~kg} \cdot \mathrm{m}^{-3}$ the air density; and $\mathrm{I}(\mathrm{I}=\mu \mathrm{A})$ the discharge current. In this work, $k$ is chosen $0.014 \mathrm{~m}^{-1 / 2}$ for the equal interelectrode distance [16], [17]. The velocity of ion flow in each channel is estimated $1.01 \mathrm{~m} / \mathrm{s}$ (detailed estimation can be found in [15]) which is in good agreement with the simulated result $(\sim 1 \mathrm{~m} / \mathrm{s})$. Experimentally, the velocity of ion flows is determined using four hotwires heated by a current of $0.2 \mathrm{~A}$. Figures 3(a) \& (b) present the time evolution of output voltage measured on a pair of hotwires that is installed symmetrically about the jet flow axis at $7.0 \mathrm{~mm}$ away from the nozzle (Fig. 1(a)). Experiment shows a discharge voltage of $2.5 \mathrm{kV}$ applied on pin electrodes (corresponding to a discharge current of 5.4 $\mu \mathrm{A})$ yields a sharp increment of the output voltage on hotwires. The observation also find that the rise time counted from the corona ignition to the stable detection is about $0.34 \mathrm{~s}$ as depicted in Fig. 3a. This confirms an existence of flow circulation in the device. Interestingly, the ion wind velocity is stable after an elapsed time of 10 seconds as shown in the Figs. 3(a) \& (b).

The angular rate sensing is investigated using a turntable on which the device is installed at the turntable center. As the turntable rotates, the jet flow inside the main chamber is deflected. This yields a variation of hotwire temperature which is detected by the output voltage measured on hotwires. Fig. 3(c) represents the time revolution of output voltage on hotwires when the turntable speed steps up from rest to $300 \mathrm{rpm}$ and then steps down to rest. With a sealed and closed configuration, the present device is able to avoid the effect of unexpected turbulences from surrounding atmosphere on the detection of high angular rate. The experimental observations have confirmed the output voltage is stable and repeatable at each step. After completed the testing, the difference of output voltage measured on a hotwire is about $1 \%$ as shown in Fig. 3 (c). With ion wind discharged at $2.5 \mathrm{kV}$ and hotwires heated by $0.2 \mathrm{~mA}$, a scale factor of $44 \mu \mathrm{V} / \mathrm{rpm}(7.3 \mu \mathrm{V} / \mathrm{o} / \mathrm{s})$ for the angular rate sensing is found. Although experimental results are not perfectly linear because of noises by the system which are not completed removed or compensated, they are quasi-linearly fitted with a coefficient of 0.98 as shown in Fig. 3(d). The sensitivity of the present gyroscope can be comparable with gas gyroscopes using the similar hotwire anemometry as published in [18]. Hence, it is feasible to improve the present device's sensitivity to order of $\mathrm{mV} / \mathrm{o} / \mathrm{s}$ using a conventional amplifier in order to achieve the performance of typical commercial counterparts [19] [20].

It is worth noting that since a humidity variation affects the corona discharge, vapour inside the sensor must be eliminated before packing the device [21]-[23]. Furthermore, gases with high conductivity (such as Helium) used in the gyroscope are preferable for the heat transfer whereas the electronegative gases $\left(\mathrm{O}_{2}, \mathrm{SF} 6\right)$ are better choices for the ion corona discharge [24]. Thus, a cautious study and relevant recalibration are required for a gas mixture used in the gyroscope. Finally, a compensation circuit with external temperature sensing element would be necessary to mitigate the effect of temperature drift [18].

\section{CONCLUSIONS}

We have reported for the first time the simulation and experimental analysis of the new jet flow based gyroscope. The device is developed using pin-ring ion wind corona discharge method in which a master ring is introduced into the new configuration to stabilize ion flow during the corona discharging. In the gyroscope, the deflection of jet flow caused by Coriolis force induced from the angular rate is detected with a sensitivity of $7.3 \mu \mathrm{V} / \%$ s. The present device is robust owing not to require any vibrating component in the new structure. Furthermore, due to low energy consumption, a small battery can be set up in the present gyroscope. Finally, this work is a valuable contribution in developing new sensing techniques and fluidics for several multidisciplinary fields such as microfluidics, analytical chemistry, and "lab-on-a-chip". 


\section{REFERENCES}

[1] B. Chua, A. S. Wexler, N. C. Tien, D. A. Niemeier, and B. A. Holmen, "Design, fabrication, and testing of a microfabricated Corona Ionizer," J. Microelectromechanical Syst., vol. 17, no. 1, pp. 115-123, 2008. DOI: 10.1109/JMEMS.2007.909515

[2] T. C. Corke, C. L. Enloe, and S. P. Wilkinson, "Dielectric Barrier Discharge Plasma Actuators for Flow Control," Annu. Rev. Fluid Mech., vol. 42, no. 1, pp. 505-529, Jan. 2010. DOI: 10.1146/annurev-fluid-121108-145550

[3] C. Kim, K. C. Noh, S. Y. Kim, and J. Hwang, "Electric propulsion using an alternating positive/negative corona discharge configuration composed of wire emitters and wire collector arrays in air," Appl. Phys. Lett., vol. 99, no. 11, pp. 2013-2016, 2011. DOI: $10.1063 / 1.3636409$

[4] V. T. Dau, T. T. Bui, T. X. Dinh, and T. Terebessy, "Pressure sensor based on bipolar discharge corona configuration," Sensors Actuators A Phys., vol. 237, pp. 81-90, 2016. DOI: 10.1146/annurev-fluid-121108-145550

[5] A. K. Sen, J. Darabi, and D. R. Knapp, "Design, fabrication and test of a microfluidic nebulizer chip for desorption electrospray ionization mass spectrometry," Sensors Actuators, B Chem., vol. 137, no. 2, pp. 789-796, 2009. DOI: $10.1016 /$ j.snb.2009.02.002

[6] V. T. Dau, T. X. Dinh, C. D. Tran, T. Terebessy, T. C. Duc, and T. T. Bui, "Particle precipitation by bipolar corona discharge ion winds," J. Aerosol Sci., vol. 124, no. December 2017, pp. 83-94, 2018. DOI: 10.1016/j.jaerosci.2018.07.007

[7] P. T. Hoa, T. X. Dinh, and V. T. Dau, "Design Study of Multidirectional Jet Flow for a Triple-Axis Fluidic Gyroscope," IEEE Sens. J., vol. 15, no. 7, pp. 4103-4113, 2015. DOI: $10.1109 /$ JSEN.2015.2411631

[8] V. T. Dau, T. X. Dinh, T. T. Bui, and C. D. Tran, "Vortex flow generator utilizing synthetic jets by diaphragm vibration," Int. J. Mech. Sci., vol. 142-143, no. May, pp. 432-439, 2018. DOI: $10.1016 /$ j.ijmecsci.2018.05.028

[9] K. Takemura, S. Yokota, M. Suzuki, K. Edamura, H. Kumagai, and T. Imamura, "A liquid rate gyroscope using electro-conjugate fluid," Sensors Actuators A Phys., vol. 149, no. 2, pp. 173-179, 2009. DOI: 10.1016/j.sna.2008.12.004

[10] J. Bahari, R. Feng, and A. M. Leung, "Robust MEMS Gyroscope Based on Thermal Principles," J. Microelectromechanical Syst., vol. 23, no. 1, pp. 100-116, Feb. 2014. DOI: 10.1109/JMEMS.2013.2262584

[11] V. T. Dau, T. X. Dinh, C. D. Tran, P. N. Bui, D. D. Vien, and H. T. Phan, "Fluidic mechanism for dual-axis gyroscope," Mech. Syst. Signal Process., vol. 108, pp. 7387, 2018. DOI: 10.1016/j.ymssp.2018.02.017

[12] Y. Fukatsu, E. Nomura, and K. Matsu, "Gas rate gyro," US4941353, 1990.

[13] Y. Cai and Y. Zhao, "Ion discharge gyroscope," US8146423, 2012.

[14] T. X. Dinh, D. B. Lam, C.-D. Tran, T. T. Bui, P. H. Pham, and V. T. Dau, "Jet flow in a circulatory miniaturized system using ion wind," Mechatronics, vol. 47, no. September, pp. 126-133, Nov. 2017. DOI: 10.1016/j.mechatronics.2017.09.007
[15] M. Robinson, "Movement of air in the electric wind of the corona discharge," Trans. Am. Inst. Electr. Eng. Part I Commun. Electron., vol. 80, no. 2, pp. 143-150, 1961. DOI: $\underline{\text { 10.1109/TCE.1961.6373091 }}$

[16] V. T. Dau, T. X. Dinh, C. D. Tran, T. T. Bui, and H. T. Phan, "A study of angular rate sensing by corona discharge ion wind," Sensors Actuators, A Phys., vol. 277, pp. 169180, 2018. DOI: $10.1016 /$ j.sna.2018.05.021

[17] L. Li, S. J. Lee, W. Kim, and D. Kim, "An empirical model for ionic wind generation by a needle-to-cylinder dc corona discharge," J. Electrostat., vol. 73, pp. 125-130, 2015. DOI: $10.1016 /$ j.elstat.2014.11.001

[18] V. T. Dau, D. V. Dao, T. Shiozawa, H. Kumagai, and S. Sugiyama, "Development of a dual-axis thermal convective gas gyroscope," J. Micromechanics Microengineering, vol. 16, no. 7, pp. 1301-1306, 2006. DOI: $10.1088 / 0960-1317 / 16 / 7 / 026$

[19] "LPY5150AL" (STMicroelectronics) [Online]. Available: https://media.digikey.com/pdf/Data Sheets/ST Microelectronics PDFS/lpy5150al.pdf.

[20] “ADXRS649 (Analog Devices)." [Online]. Available: https://www.analog.com/media/en/technicaldocumentation/data-sheets/ADXRS649.pdf.

[21]B. R. Maskell, "The effect of humidity on a corona discharge in air," Royal Aircraft Establishment, Farnborough, U.K., Tech. Rep., p. 70106, 1970.

[22]X. Wang and C. You, "Effect of humidity on negative corona discharge of electrostatic precipitators," IEEE Trans. Dielectr. Electr. Insul., vol. 20, no. 5, pp. 17201726, 2013. DOI: 10.1109/TDEI.2013.6633702

[23] V. T. Dau, T. X. Dinh, T. T. Bui, and T. Terebessy, "Bipolar corona assisted jet flow for fluidic application," Flow Meas. Instrum., vol. 50, pp. 252-260, 2016. DOI: 10.1016/j.flowmeasinst.2016.07.005

[24] D. Rafalskyi, L. Popelier, A. Aanesland, D. Rafalskyi, L. Popelier, and A. Aanesland, "Experimental validation of the dual positive and negative ion beam acceleration in the plasma propulsion with electronegative gases thruster," $J$. Appl. Phys., vol. 115, p. 053301, 2014. DOI: $\underline{10.1063 / 1.4863876}$ 